\title{
Structure and Dynamics of Nanocatalysts
}

\author{
S. Helveg* \\ * Haldor Topsøe A/S, Nymøllevej 55, DK-2800 Kgs. Lyngby, Denmark
}

Heterogeneous catalysis plays an important role in diverse areas such as environmental protection, energy conversion and supply of chemicals. The catalysts typically consist of nanometer-sized particles dispersed on high-surface area support materials, and the catalytic effect, converting reactants into product molecules, is mediated at the surfaces of the nanoparticles (nanocatalysts). The properties of the active surface sites depend among others on the type, location and number of surrounding surface atoms, the support materials and the presence of adsorbed reactants and products. A detailed description of the structure and reactivity of the nanocatalysts is therefore of utmost importance to aid a rational design of nanocatalysts with improved performance. Although nanocatalysts may be synthesized with a predefined shape and structure, there is, however, no guarantee that the as-synthesized nanocatalysts remain stable when subjected to operation conditions. The realization that the structure and properties of the nanocatalysts may intimately depend on the reaction conditions has spurred a tremendous interest for also characterizing the atomic-scale structural and chemical state of the nanocatalysts in situ during exposure to reaction conditions [1]. High-resolution transmission electron microscopy (HRTEM) has proved to be a powerful technique that allows unique information about the size, shape and structure of nanocatalysts to be obtained at the atomic level. The HRTEM examinations of nanocatalysts are typically conducted under the high vacuum conditions $\left(10^{-9} \mathrm{bar}\right)$ in the microscopes. When used for in situ studies of gas-solid interactions the spatial resolution is hampered by the introduction of gas atoms, which scatter the electron beam and blur the object details. Limitation of the number of gas atoms is therefore a key requirement. This can be reached by limiting the pressure and by confining the gas along the path of the beam into a layer that is as thin as possible. Confinement has been attained by two methods: by differentially pumped vacuum systems (also referred to as Environmental Transmission Electron Microscopy) and by windowed cells [2,3]. Both methods have provided HRTEM images of nanocatalysts with atomic lattice fringes with spacing smaller than $0.2-0.3 \mathrm{~nm}$ at pressures up to about $10^{*} 10^{-3}$ bar. Recently, a nanoreactor was introduced as a micro-electro-mechanical system and functionalized for in situ HRTEM imaging of nanocatalysts during exposure to gasses at ambient pressures up to ca. 1 bar and temperatures up to ca. $500^{\circ} \mathrm{C}[4,5]$. These in situ HRTEM capabilities provide unprecedented new insight into the structure and dynamics of nanocatalysts. Specifically, information can be obtained about the exposed surface sites and support interactions as well as about mechanisms and kinetics involved in surface processes induced by the reactive gas environment. In this contribution, I will outline recent developments and applications of in situ HRTEM for studies of industrially important nanocatalyst systems, e.g. [3-10]. Furthermore, I will discuss how in situ HRTEM observations can be used beneficially in combination with information from theoretical methods, based on e.g. Density Functional Theory, and from complementary in situ characterization techniques to elucidate the role of gas-surface interactions on the working nanocatalysts.

References

[1] A.M. Molenbroek, Top. Catal. 52 (2009) 1303.

[2] P.L. Gai et al., MRS Bulletin 32 (2007) 1044.

[3] P.L. Hansen, S. Helveg, A.K. Datye, Adv. Catal. 50 (2006) 77.

[4] J.F. Creemer et al., Ultramicroscopy 108 (2010) 993.

[5] J.F. Creemer et al., J. MEMS (2010) in press.

[6] P.L. Hansen, Science 295 (2002) 2053.

[7] P. Vesborg et al., J. Catal. 262 (2009) 65.

[8] S. Helveg et al., Nature 427 (2004) 426.

[9] F. Abild-Pedersen, Phys. Rev. B 73 (2006) 115419.

[10] S. B. Simonsen et al., J. Catal. 255 (2008) 1. 